\section{International Scientific Journal Theoretical \& Applied Science}

\author{
p-ISSN: 2308-4944 (print) e-ISSN: 2409-0085 (online) \\ Year: 2015 Issue: 11 Volume: 31 \\ Published: $30.11 .2015 \quad$ http://T-Science.org
}

SECTION 4. Computer science, computer engineering and automation.
Victor Aleksandrovich Melent'ev

Philosophy Doctor, senior research associate

Rzhanov Institute of Semiconductor Physics Siberian Branch of Russian Academy of Sciences (ISP SB RAS) melva@isp.nsc.ru

Valery Ivanovitsh Shubin

Head of Department of Computer Networks ISP SB

RAS

woland@isp.nsc.ru

Anatolij Filippovich Zadorozhny

Philosophy Doctor, Head of Department of Information Systems and Technology, Novosibirsk State University of Architecture and Civil

Engineering (Sibstrin)

3af@ngs.ru

\title{
TOPOLOGICAL SCALABILITY OF HYPERCUBIC PARALLEL SYSTEMS AND TASKS
}

Abstract: We research the potential parallelism of hypercubic computing systems, which is limited by the maximal allowable distance between processors, which are adjacent on data. Functions of a upper limitation on parallelism and of a topological scalability of such systems in case of solving tasks with complete graph on data, and also of tasks with ring and star topology on data are formalized. The corresponding diagrams are given and their analysis is given.

Key words: parallel systems and tasks, scalability, hypercubic topology, the ring and star topologies.

Language: Russian

Citation: Melent'ev VA, Shubin VI, Zadorozhny AF (2015) TOPOLOGICAL SCALABILITY OF HYPERCUBIC PARALLEL SYSTEMS AND TASKS. ISJ Theoretical \& Applied Science 11 (31): 122-129.

Soi: http://s-o-i.org/1.1/TAS-11-31-19 Doi: crossef http://dx.doi.org/10.15863/TAS.2015.11.31.19

\section{ТОПОЛОГИЧЕСКАЯ МАСШТАБИРУЕМОСТЬ ГИПЕРКУБИЧЕСКИХ ПАРАЛЛЕЛЬНЫХ СИСТЕМ И ЗАДАЧ}

Аннотация: Исследуется потенциальный параллелизм гиперкубических вычислительных систем, лимитированный предельно допустимым расстоянием между информационно смежными в задаче процессорами. Формализованы функции предельной распараллеливаемости и топологической масштабируемости таких систем при реализаџии информаџионно полносвязных задач, а также задач с кольцевой и звездной информационной топологией. Приведены соответствующчие графики функций, и дан их анализ.

Ключевые слова: параллельные системы и задачи, масштабируемость, гиперкубическая топология, кольцевая и звездная топологии.

Работа выполнена при поддержке Российского фонда фундаментальньх исследований (проект 14-07-00169a)

\section{Введение}

Известно, что ускорение и эффективность распараллеливания задачи определяются самым неэффективным компонентом реализующей ее подсистемы, каковым при отсутствии в задаче скалярных фрагментов [1, с. 484] является коммуникационная среда вычислительной системы (BC), удельное (на единицу информации) быстродействие которой обусловлено используемой сетевой технологией

\section{и}

расстояниями

между информационно-смежными в задаче процессорами [2, с. 2]. Центральной проблемой горизонтального масштабирования ВС [3] и ожидаемого в связи с этим изменения потенциала параллелизма решаемых задач является то, что увеличение порядка информационного графа задачи приводит к неполиномиальному росту трудоемкости поиска изоморфных его вложений при существенном снижении вероятности 
наличия таковых. Последнее связано с тем, что используемые при вложении параллельной задачи алгоритмы основаны, как правило, на сопоставлении отношений смежности вершин информационного графа и вершин графа ВС. Однако, в силу известных технико-экономических факторов, полная связность всех процессоров системы реализована быть не может, а условие физической смежности задействованных в параллельной задаче процессоров не всегда может быть реализовано даже при малом их числе, масштабирование же задачи лишь усугубляет эту ситуацию.

В [4, с. 213] информационную модель ВС предложено представлять графами, отношения смежности вершин в которых удовлетворяют допускаемым размещаемыми подсистемами требованиям в части предельно допускаемых расстояний между ними. В [5] установлены функциональные зависимости предельно-допускаемых расстояний $\partial$ между информационно-смежными процессорами от присущих задаче $W_{p}$ объемов вычислительных операций $W$ и обмениваемых данных $Q$, от требуемых значений ускорения $S_{p}$ и/или эффективности $E_{p}$ решения этих задач при их распараллеливании на $p$ ветвей в системе с определенной сетевой технологией (Network Technology-NT). Бо́льшим значениям $\partial$ при этом соответствуют бо́льшие значения степеней $s$ получаемых таким образом графов $G_{\partial}$ д-достижимости и бо́льшие значения их плотностей ${ }^{1} \quad \varphi\left(G_{\partial}\right) \equiv \varphi_{\partial}(G) . \quad$ Естественно, что вложение в такие графы параллельных задач, ранг которых не превышает $\partial$-плотности, может быть произвольным, а возможности изоморфного вложения в них задач с бо́льшим числом ветвей - существенно выше.

В [6, с. 23-26] проведено исследование функциональной зависимости порядка наибольшей компоненты д-достижимости ( $\partial$ клики) гиперкубического графа ВС от его размерности и от лимитируемой достижимости $\partial$ его вершин.

В работе [5] впервые предпринята попытка восполнить имеющийся пробел в оценке влияния топологии на масштабируемость параллельных систем и решаемых на них задач. С этой целью была предложена разделенная на две составляющие модель параллельных вычислений: 1-я отнесена к параллельным приложениям и приписывает им свойства неограниченной распараллеливаемости [7, с. 198], 2-я отнесена к вычислительной системе, ограничения параллелизма в которой обусловлены дефицитным в сравнении с процессорами быстродействием коммуникационной среды.

Это позволило разделить технологический и топологический аспекты масштабирования ВС и исследовать взаимную обусловленность соответствующих архитектурных компонентов в масштабировании задач. При этом выявлено, что в зависимости от присущих задаче объемов вычислительных операций $W$ и обмениваемых данных $Q$ ускорение $S$ ее решения предельно-допускаемое расстояние $\partial$ между информационно-смежными процессорами и минимальное число $p$ используемых при этом процессоров коррелированы используемой в системе NT. Совместность же этих, определяемых сетевой технологией, параметров зависит уже от топологии ВС. Иными словами, если при решении $S(W, Q)$-задачи в ВС заданное ускорение может быть обеспечено при ее разбиении не менее чем на $p$ параллельных ветвей, причем расстояния между информационно смежными процессорами ограничены используемой NT и не должны превышать $\partial(p)$, то граф ВС должен содержать отвечающий этому условию подграф порядка $p$.

В данной работе на основе предложенных в [5] показателей проведено исследование топологической масштабируемости гиперкубических ВC при решении задач с заданной информационной топологией.

\section{Понятие \\ топологической масштабируемости параллельных систем и задач}

Понятие топологической масштабируемости параллельных систем и задач неразрывно связано с проблемой оптимального вложения вторых в первые. В работе [5] впервые введено это понятие и представлено решение данной проблемы в постановке, основанной на замене отношений смежности вершин графа ВС отношениями их достижимости; при этом вершины считаются достижимыми, если расстояние между ними не превышает порогового для рассматриваемой задачи значения $\partial$.

Полученный таким образом граф $\partial$-достижимости $G_{\partial}$ в сравнении с исходным графом $G$ вычислительной системы обладает большей степенью $s\left(G_{\partial}\right)>s(G)$ и, соответственно, меньшим диаметром $d\left(G_{\partial}\right)<d(G)^{2}$, поэтому шансы конфигурирования подсистем, в которых расстояния между информационно-смежными процессорами общим числом $p$ не превышают

1 Плотность $\varphi$ графа $G$ - число вершин максимальной по включению в нем клики. Плотность $\varphi\left(G_{\partial}\right)$ графа $\partial$-достижимости $G_{\partial}$ будем называть также $\partial$-плотностью $\varphi_{\partial}(G)$ графа $G$.

ISPC Technological advances,

${ }^{2}$ При $d(G) \leq \partial$ граф $\partial$-достижимости $G_{\partial}$ является полным с $d\left(G_{\partial}\right)=1$. 
определяемого сетевой технологией значения достижимости $\partial(p)$, существенно повышаются.

Взаимная обусловленность числа $p$ процессоров и предельного расстояния $\partial(p)$ между информационно-смежными вершинами соответствующего задаче подграфа графа $\mathrm{BC}$, а также зависимость этих величин от используемой в системе NT, от соотношения объемов вычислений $W$ и информационных взаимодействий $Q$ в параллельной $(W, Q)$-задаче и от директивных значений ускорения $S_{p}$ и/или эффективности $E_{p}$ ее решения установлены в [5]. Совместность обусловленных $N T$ значений $p$ и $\partial(p)$ в рамках набора из $N$ решаемых в ВС задач с заданными рангами $p_{i} \quad(i=\overline{1, N}) \quad$ их распараллеливания обеспечивается выбором соответствующей сетевой топологии с требуемой для каждой пары $\left\{p_{i}, \partial\left(p_{i}\right)\right\}$ степенью обеспечения этой совместности.

Максимальное по включению подмножество вершин, связанных между собой отношением Ә-достижимости, порождает клику $K\left(G_{\partial}\right)$ соответствующего графа $G_{\partial}$, иначе - - -клику $K_{\partial}(G)$ графа $G$ системы. Подобно плотности $\varphi(G)$ графа $G$, определяемой порядком его наибольшей клики $[8$, с. 21], порядок наибольшей из максимальных $\partial$-клик графа $G(V, E)$ назван плотностью $\varphi\left(G_{\partial}\right)$ графа $\partial$-достижимости, или Ә-плотностью $\quad \varphi_{\partial}(G) \quad$ графа $\quad G(V, E) \quad-$ $\varphi\left(G_{\partial}\right) \equiv \varphi_{\partial}(G)$.

Известно [5], что порядок $n$ максимальной в графе ВС $\partial$-клики $K_{\partial}(G)$, все вершины которой по определению взаимно д-достижимы, или $\partial$-плотность $\varphi_{\partial}(G)$ этого графа $\left(n\left(K_{\partial}(G)\right) \equiv \varphi_{\partial}(G)\right)$ ограничивает сверху параллелизм (число ветвей) только для информационно-полносвязных задач. Для задач же, в которых информационный граф $W_{p}$ задачи с числом ветвей $p$ обладает плотностью $\varphi\left(W_{p}\right)$, меньшей $p$, верхние границы могут быть бо́льшими $\varphi_{\partial}(G)$ в случае, если в графе $\partial$-достижимости $G_{\partial}$ существует подграф порядка $p>\varphi_{\partial}(G)$, изоморфный графу $W_{p}$. Таким образом, пределы распараллеливания решаемых в системе задач в зависимости от допускаемой для каждой из них достижимости $\partial$ находятся в диапазонах от $\varphi_{\partial}(G)$ до $n(G)$. И чем у́же эти диапазоны, т. е. чем больше значение $\partial$-плотностей, тем большим потенциалом в отношении масштабирования обладает используемая в ВС топология.

Так как в наборе $(W, Q)$-задач, решаемых в исследуемой ВC с известной для нее $N T$, каждая из задач может быть классифицирована по соответствующим ей значениям предельно-допускаемой достижимости $\partial(p)$, функцию масштабируемости ВС определяем для присущих ее графу $G$ достижимостей $1 \leq \partial \leq d(G)$ от единичного значения до диаметра $d(G)$, и измеряем эту функцию $\mu_{\partial}(G)$ нормированной масштабируемым порядком $n(G)$ графа $\mathrm{BC}$ плотностью $\varphi_{\partial}(G): \mu_{\partial}(G)=\varphi_{\partial}(G) / n(G)$.

Топологическая гиперкубических ВС

масштабируемость

Пусть ориентация гиперкубической ВС с используемой ею $N T$ определена перечнем распараллеливаемых пользовательских приложений, каждое из которых отвечает условиям неограниченной распараллеливаемости [7, с. 198], т. е. не содержит скалярных фрагментов и характеризуется объемами (или диапазонами изменения) $W$ и $Q$ вычислительных и обменных операций, равномерно распределяемыми между процессорами подсистемы в зависимости от числа параллельных ветвей $p$. Тогда согласно предложенной в [5] модели существуют предельные значения допускаемых для каждой из этих задач достижимостей, и все решаемые в исследуемой ВС пользовательские задачи могут быть классифицированы в соответствии с этими значениями $\partial$. В таком случае выбор топологии, адекватной перечню решаемых в системе с заданной NT задач может быть обоснован анализом функций $\partial$-плотности сопоставляемых топологий на соответствующем этим задачам диапазоне $\partial_{\min } \leq \partial \leq \partial_{\max }$ изменения достижимостей. Ниже дадим такой анализ для ВС с гиперкубической топологией.

В работе [6, с. 23-26] проведено исследование и определена формальная зависимость порядка $n_{\partial}\left(H_{s}\right)$ максимальной по включению Ә-компоненты $s$-мерного куба $H_{s}$, или, что равнозначно, $\partial$-плотности $\varphi_{\partial}\left(H_{s}\right) \equiv n_{\partial}\left(H_{s}\right)$ от предельно-допускаемого расстояния $\partial$ между информационно-смежными его вершинами:

$$
\varphi_{\partial}\left(H_{S}\right)=\left\{\begin{array}{cr}
\sum_{i=0}^{\partial / 2}\left(\begin{array}{l}
S \\
i
\end{array}\right) & \text { четн. } \partial, \\
\left(\begin{array}{c}
s-1 \\
\lfloor\partial / 2\rfloor
\end{array}\right)+\sum_{i=0}^{\lfloor\partial / 2\rfloor}\left(\begin{array}{l}
S \\
i
\end{array}\right) & \text { нечетн. } \partial .
\end{array}\right.
$$

Соответствующий (1) график функции Ә-плотности гиперкуба от его степени $s$ представлен на рис. 1 .

В связи с тем, что масштабирование гиперкуба не может быть «непрерывным» и кратно двум, оси графика Ә-плотности выполнены в логарифмическом масштабе с двоичным основанием. При этом, несмотря на дискретность масштабирования общего числа процессоров, точки на графике, соответствующие значениям функции д-плотности $\varphi_{\partial}\left(H_{s}\right)$, соединены для лучшего восприятия их приписываемости значениям достижимости $\partial$.

Хотя $\partial$-плотность графа вычислительной системы характеризует предельные возможности распараллеливания
B ней 


\begin{tabular}{|c|c|c|c|c|c|c|}
\hline Impact Factor: & $\begin{array}{l}\text { ISRA (India) } \\
\text { ISI (Dubai, UAE } \\
\text { GIF (Australia) } \\
\text { JIF }\end{array}$ & $\begin{array}{l}=1.344 \\
=0.829 \\
=0.356 \\
=1.500\end{array}$ & $\begin{array}{l}\text { SIS (USA) = } \\
\text { PИHЦ (Russia) = } \\
\text { ESJI (KZ) = } \\
\text { SJIF (Morocco) }\end{array}$ & $\begin{array}{l}=0.912 \\
=0.179 \\
=1.042 \\
=\mathbf{2 . 0 3 1}\end{array}$ & ICV (Poland) & $=6.630$ \\
\hline
\end{tabular}

информационно-полносвязных задач, эта величина, не являясь количественной характеристикой таких возможностей ВС при реализации в ней любых других, не требующих информационной полносвязности, задач, определяет нижнюю границу их параллелизма и, таким образом, служит качественной (в отношении лучше-хуже) оценкой потенциала системы в их параллельной реализации. Количественную оценку масштабируемости гиперкубической $\mathrm{BC}$, связанную с топологией решаемой задачи рассмотрим в следующем разделе.

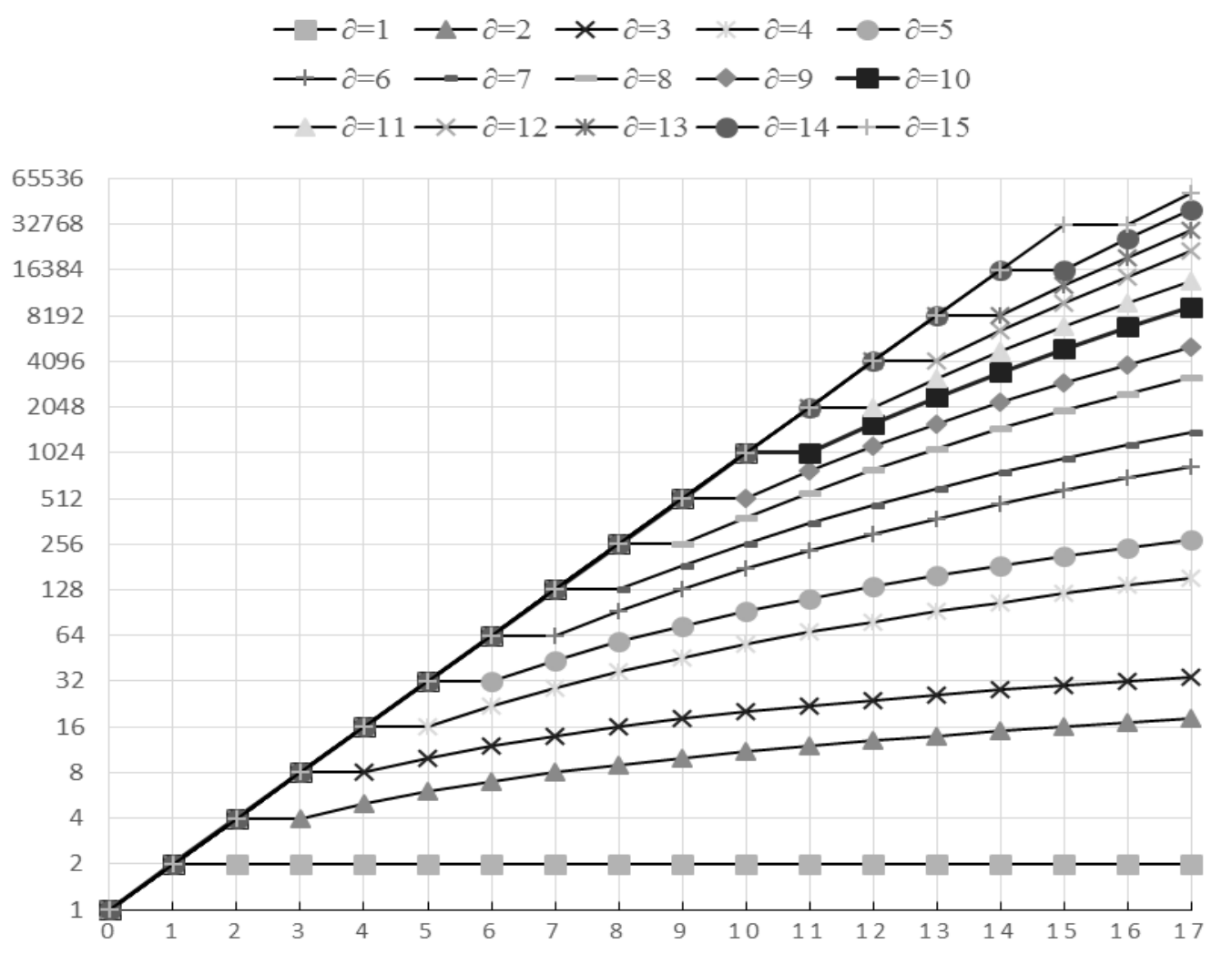

Рисунок 1 - Семейство функций $\partial$-плотности гиперкуба $\varphi_{\partial}\left(H_{s}\right)=n_{\partial}\left(K, H_{s}\right)$.

Из рис. 1 видно, что каждая из кривых, соответствующих обусловленным задачами и применяемой в ВС NT значениям достижимости $\partial$, имеет три характерных участка: 1-й участок соответствует прямопропорциональному росту $\partial$-плотности $\quad-\quad s<\partial \Rightarrow \Delta \varphi_{\partial}\left(H_{s}\right) / \Delta p=1$; приращение $\partial$-плотности на участке от $s=\partial$ до $s=\partial+1 \quad$ является нулевым $s=\partial \Rightarrow \varphi_{\partial}\left(H_{s+1}\right)-\varphi_{\partial}\left(H_{s}\right)=0 ; 3$-й участок с $s>\partial$, характеризуется существенным замедлением роста функции Ә-плотности сравнении с 1-м участком $-s>\partial \Rightarrow 0<\Delta \varphi_{\partial}\left(H_{s}\right) / \Delta p<1$ и функция $\varphi_{\partial}\left(H_{s}\right)$ теряет свойство линейности.

Отметим еще одну особенность 3-го участка: реализуемые в системе задачи, допускающие бо́льшие межпроцессорные расстояния, не только обладают бо́льшим в абсолютном выражении потенциальным параллелизмом вследствие того, что $s>\partial_{2}>$ $\partial_{1} \Rightarrow \varphi_{\partial_{2}}\left(H_{s}\right)>\varphi_{\partial_{1}}\left(H_{s}\right)$, 一 им соответствует и большее, при единичном приращении $s$, приращение $\partial$-плотности. Если при этом значение $\partial$ является четным $(\partial=2 x \leq s-1$, где $x$ - целое число), то $\varphi_{\partial+2}\left(H_{s}\right)-\varphi_{\partial+1}\left(H_{s}\right)>$ $\varphi_{\partial+1}\left(H_{s}\right)-\varphi_{\partial}\left(H_{s}\right)$.

Переведя вышесказанное к интересующей нас масштабируемости гиперкубической $\mathrm{BC}$, из приведенного на рис. 2 семейства функций $\mu_{\partial}\left(H_{s}\right)$ масштабируемости гиперкубической ВС можно увидеть, что масштабируемость гиперкубической ВC на участке с $s \leq \partial$ не зависит от информационной топологии распараллеливаемых задач, является максимальной и равна единице $s \leq \partial \Leftrightarrow \mu_{\partial}\left(H_{s}\right)=1$. Это единичное значение функции $\mu_{\partial}\left(H_{s}\right)$ является верхней границей диапазона топологической масштабируемости гиперкубической ВС на множестве решаемых в ней задач вне зависимости от размерности $s$ гиперкуба и от заданных для задач значений $\partial$. Дальнейшее $(s>\partial)$ наращивание числа измерений гиперкубической ВС сопровождается расширением вниз диапазона топологической масштабируемости, за счет значений, соответствующих решению информационно-полносвязных задач.

ISPC Technological advances, 


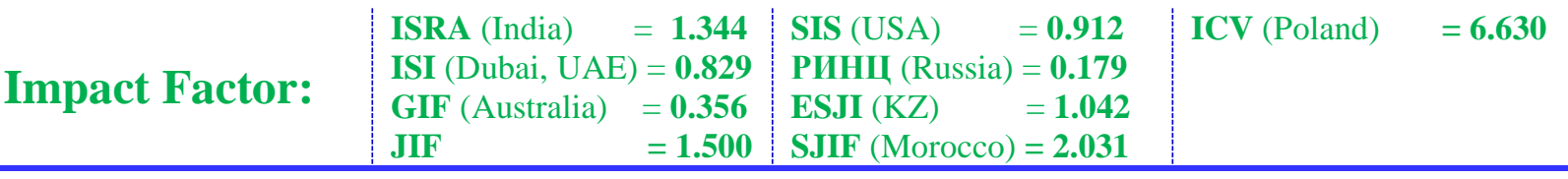

Топологическая масштабируемость гиперкубических ВС при реализации типовых коммуникационных схем параллельных задач

В [5] предложен показатель топологической масштабируемости задач

$$
\mu\left(W_{p}\right)=\frac{\left(p-\varphi\left(W_{p}\right)\right) \cdot\left(p-s\left(W_{p}\right)\right)}{p^{2}},
$$

учитывающий плотность $\varphi\left(W_{p}\right)$ и степень $s\left(W_{p}\right)$ информационного графа $W_{p}$ задачи ранга $p$, решаемой в системе на $p$ процессорах.

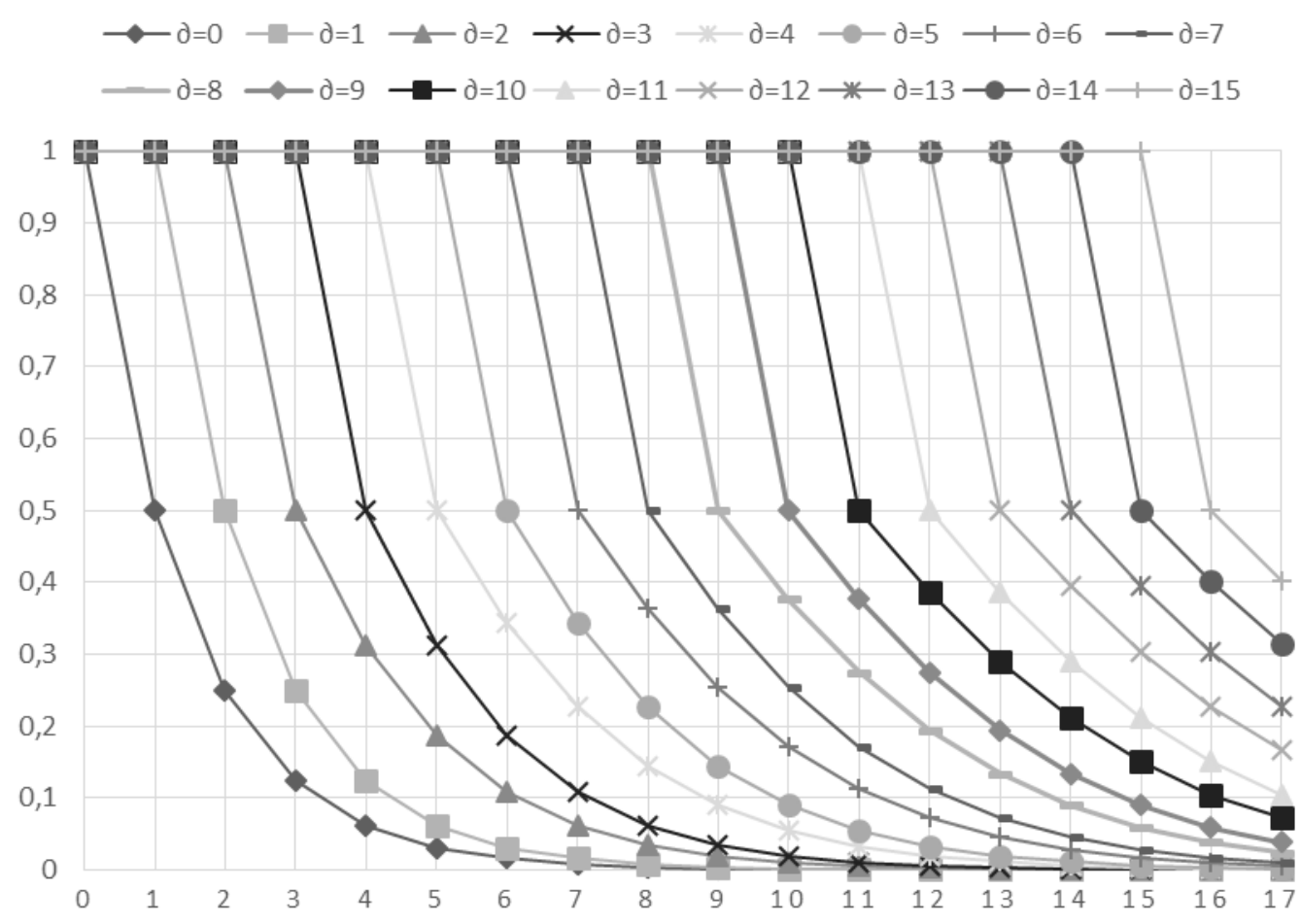

Рисунок 2 - Семейство функций топологической масштабируемости гиперкубической ВС.

Заметим, что функция $\mu\left(W_{p}\right)$ характеризует топологический аспект масштабирования ${ }^{3}$ только задачи - она абстрагирована и от топологии используемой для ее решения системы, и от предельно-допускаемого расстояния между информационно-смежными процессорами: чем ближе значение функции к единичному, тем эффективнее может быть размещена эта задача в произвольной ВС при заданном $p$.

Здесь мы поставим задачу оценить возможности вычислительной системы с заданной топологией в решении задач, параллельная реализация которых предполагает использование некоторой информационной топологии. Итак, топология ВС задана графом $G$ порядка $n$, информационная топология задачи задана графом $W$, предельно-допускаемое расстояние между информационно-смежными процессорами определено некоторым значением

достижимости $\partial \geq 1$. Для определения масштабируемости $\mu_{\partial}(W, G) \equiv \mu\left(W, G_{\partial}\right) \quad$ данной системы в параллельной реализации рассматриваемой задачи при заданном для нее значении $\partial$ следует определить порядок $n\left(W, G_{\partial}\right) \equiv n_{\partial}(W, G)$ максимального по включению изоморфного графу $W$ подграфа в графе $\partial$-достижимости $G_{\partial}$, при этом топологическая масштабируемость такой ВС с $G$-топологией при решении на ней задач $\mathrm{c}$ информационной $W$-топологией определится отношением $\mu_{\partial}(W, G)=n_{\partial}(W, G) / n(G)$. Как мы уже выяснили выше, для задач с полносвязной топологией (по аналогии с традиционным обозначением полного графа через $K_{n}$ обозначим их топологию $W$ через $K-\mu_{\partial}(K, G)=n_{\partial}(K, G) / n(G) \equiv \mu_{\partial}(G) . \quad$ И, как показано в предыдущем разделе, эта функция ограничивает снизу область определения функции масштабируемости $\mathrm{BC} \mathrm{c}$ заданной топологией для любой из множества решаемых в ней задач.

3 Здесь имеется в виду масштабирование задачи по числу параллельных ветвей.

ISPC Technological advances, 


\begin{tabular}{|c|c|c|c|c|c|c|}
\hline Impact Factor: & $\begin{array}{l}\text { ISRA (India) } \\
\text { ISI (Dubai, UAF } \\
\text { GIF (Australia) } \\
\text { JIF }\end{array}$ & $\begin{array}{r}=1.344 \\
=0.829 \\
=0.356 \\
=1.500\end{array}$ & $\begin{array}{l}\text { SIS (USA) } \\
\text { PИНЦ (Russia) } \\
\text { ESJI (KZ) } \\
\text { SJIF (Morocco) }\end{array}$ & $\begin{array}{l}=0.912 \\
=0.179 \\
=1.042 \\
=2.031\end{array}$ & ICV (Poland) & $=6.630$ \\
\hline
\end{tabular}

Рассмотрим введенную выше функцию для гиперкубических $\mathrm{BC}\left(G=H_{s}, s-\right.$ размерность гиперкуба) на примере задач с некоторыми типовыми информационными топологиями [9, c. 376]. Известно [10, с. 32], что гиперкуб обладает свойством гамильтоновости, т. е. содержит цикл, проходящий через каждую вершину ровно один раз. Следовательно, для задач с кольцевой топологией $(W=C)-$ $n_{\partial}\left(C, H_{s}\right)=2^{s}, \quad$ поэтому $\quad \forall \partial \geq 1 \mu_{\partial}\left(C, H_{s}\right)=1$. Совпадение значений функции топологической масштабируемости $\mu_{\partial}\left(C, H_{s}\right)$ с единицей даже для $\partial=1$ при любой степени $s$ гиперкуба означает, что использование этой топологии не вносит никаких ограничений в распараллеливание задач с кольцевой топологией, и такие ограничения могут быть обусловлены лишь недостаточным быстродействием используемой в $\mathrm{BC}$ сетевой технологии.

Учитывая те же рассуждения, что и используемые при выводе формулы (1) в работе [5], для задачи со звездной $(Z)$ топологией получим:

$$
\begin{array}{cc}
n_{\partial}\left(Z, H_{S}\right) & =\sum_{i=0}^{\partial}\left(\begin{array}{c}
S \\
i
\end{array}\right) . \\
\text { На } & \text { рис. } 3 \text { приведено }
\end{array}
$$
соответствующих функций. Сопоставляя графики рис. 3 и рис. 1, нетрудно заметить существенное превышение значений $n_{\partial}\left(Z, H_{s}\right)$ над $n_{\partial}\left(K, H_{s}\right) \equiv \varphi_{\partial}\left(H_{s}\right)$, например, для $\partial=1 \varphi_{\partial}\left(H_{s}\right)=2$ вне зависимости от масштабирования системы, тогда как $n_{\partial}\left(Z, H_{s}\right)$ растет с изменением $s$ от $n_{1}\left(Z, H_{1}\right)=2$ до $n_{1}\left(Z, H_{17}\right)=18$.

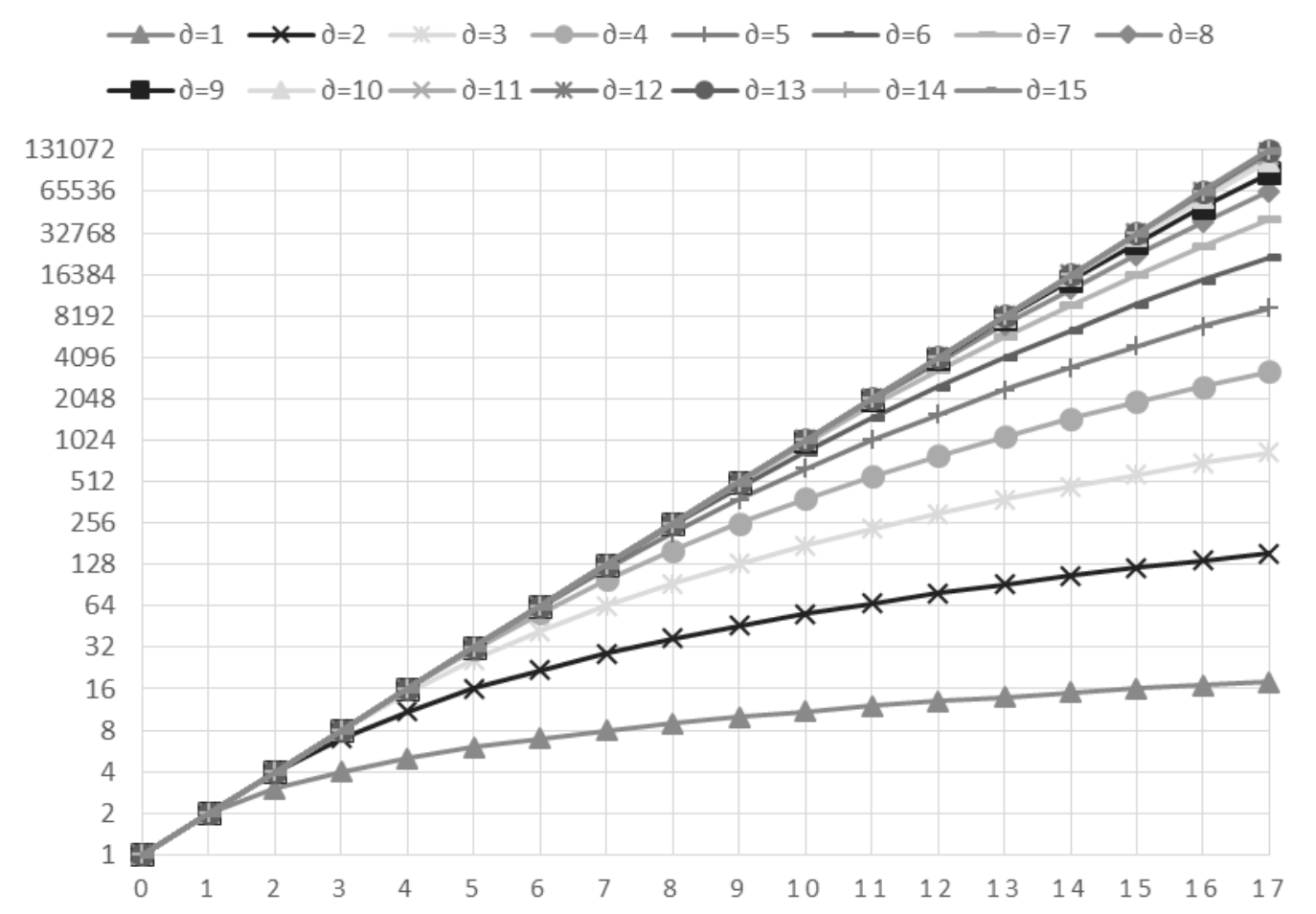

Рисунок 3 - Семейство функций максимального порядка $\mathbf{n}_{2}\left(\mathrm{Z}, \mathrm{H}_{\mathrm{s}}\right)$ «звезды» в гиперкубической $\mathrm{BC}$.

Семейство функций масштабируемости гиперкубической $\mathrm{BC}$ на задачах со звездной топологией (рис. 4) также значительно отличается от аналогичного семейства на рис. 2. Сопоставляя эти семейства видим существенное улучшение качество масштабируемости гиперкубической ВС на задачах со звездной информационной топологией. Для лучшего визуального восприятия различий в масштабируемости рассматриваемой системы на задачах с упомянутыми топологиями сведем эти функции на одном рисунке (рис. 5), оставив при этом лишь 3 значения допустимой достижимости информационно-смежных процессоров: $\partial=2$, $\partial=4$ и $\partial=7$. Представленные на этом рисунке графики наглядно демонстрируют доминирующую зависимость масштабируемости от используемой сетевой технологии: использование $N T$, позволяющей увеличить предельно-допустимое расстояние, например, в 2 раза с $\partial=2$ до $\partial=4$ в задачах со звездной топологией увеличивает коэффициент масштабируемости с 0,6875 до 1.0 при $s=4$ и с 0,34375 до 0,8906 при $s=6$. Аналогичные 


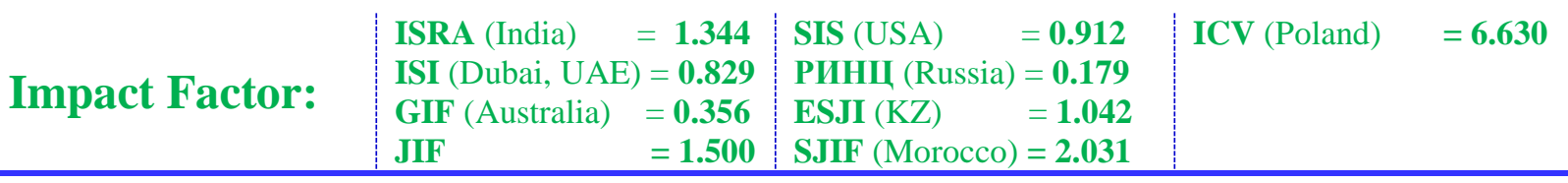

изменения масштабируемости на полносвязных задачах составляют соответственно 0,3125-1,0 и 0,109375-0,34375. Эти же графики позволяют убедиться в том, что потенциал предельного параллелизма ВC существенно зависит от топологий решаемых на ней задач.

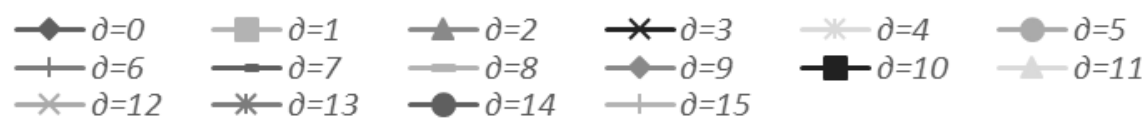

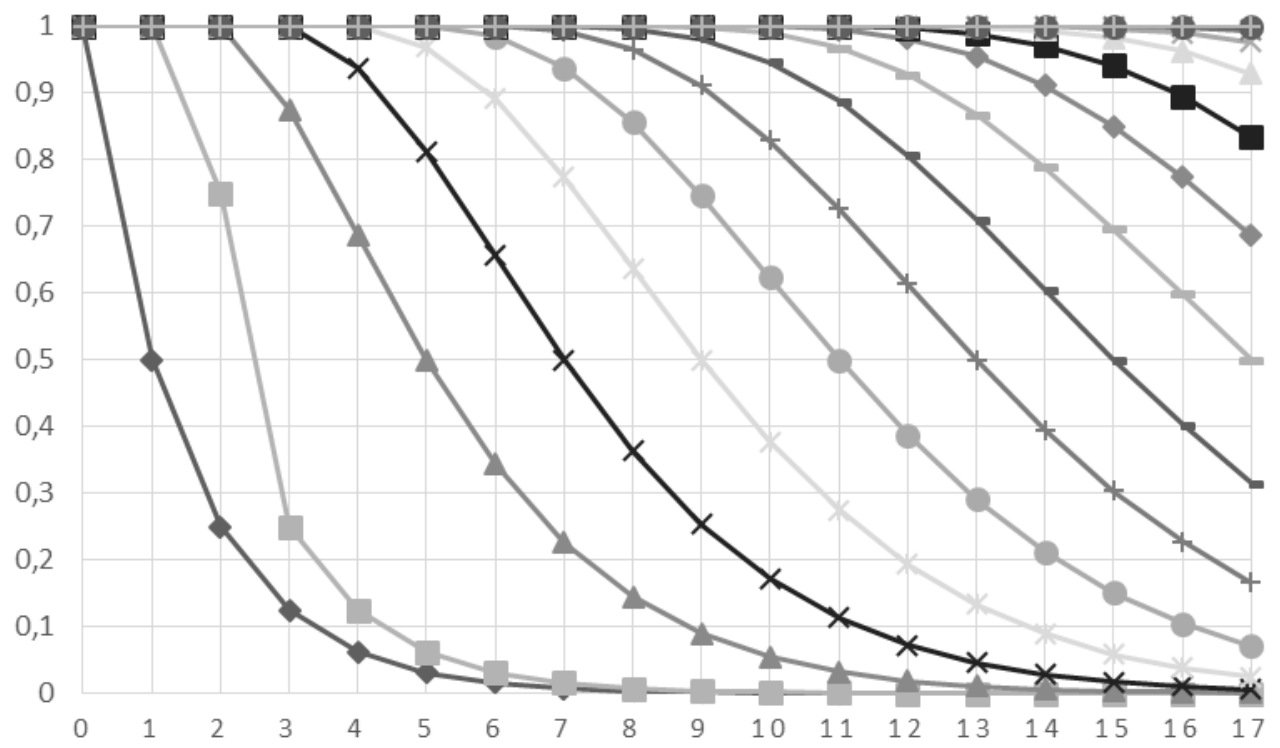

Рисунок 4 - Семейство функций масштабируемости гиперкубической ВС на задачах со звездной топологией.

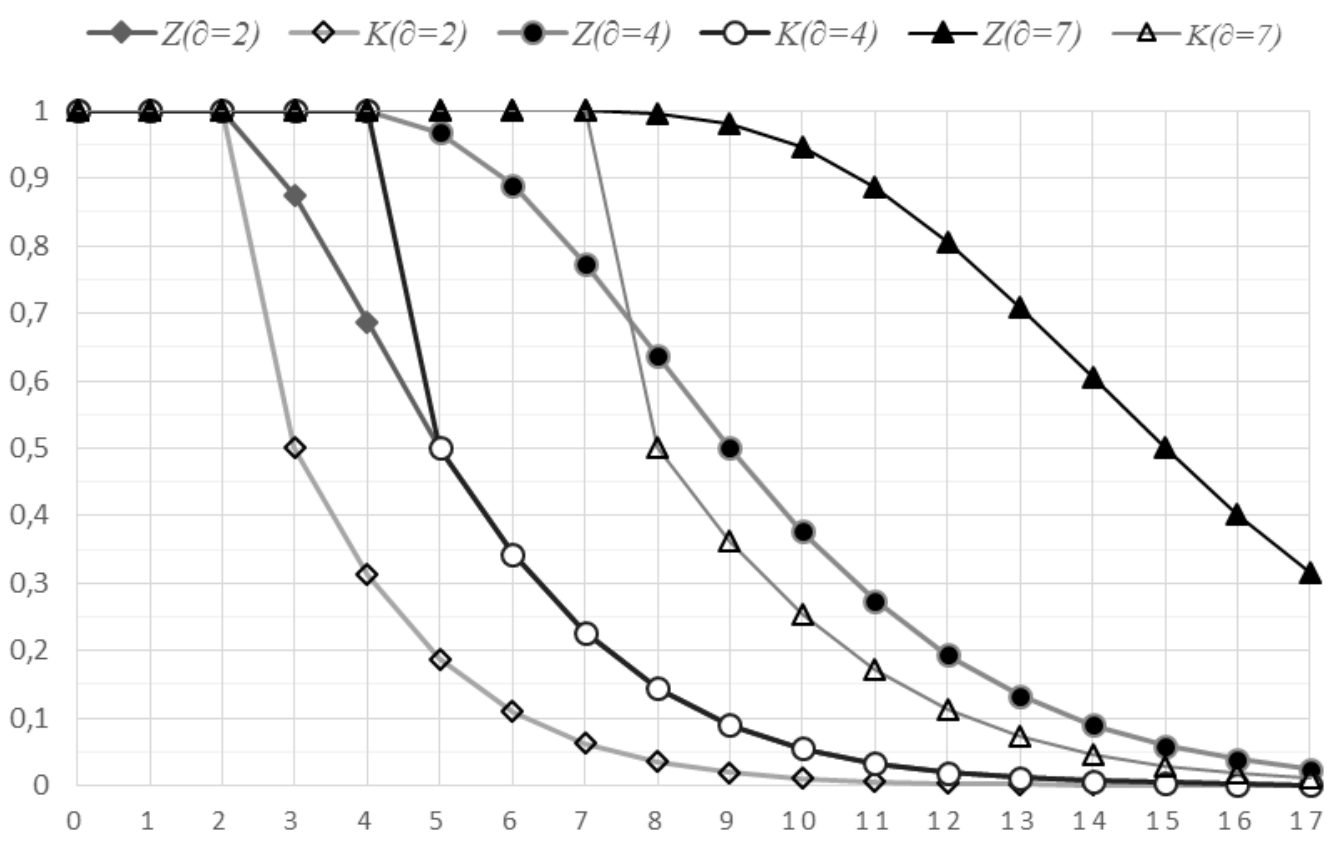

Рисунок 5 - Сопоставление функций топологической масштабируемости гиперкубической ВС на задачах с полносвязной и со звездной топологиями. 


\begin{tabular}{|c|c|c|c|c|c|}
\hline Impact Factor: & $\begin{array}{l}\text { ISRA (India) } \\
\text { ISI (Dubai, UAF } \\
\text { GIF (Australia) } \\
\text { JIF }\end{array}$ & $\begin{array}{l}=1.344 \\
=0.829 \\
=0.356 \\
=1.500\end{array}$ & $\begin{array}{l}\text { SIS (USA) } \\
\text { PИНЦ (Russia } \\
\text { ESJI (KZ) } \\
\text { SJIF (Morocco }\end{array}$ & $\begin{array}{l}=0.912 \\
=0.179 \\
=1.042 \\
=2.031\end{array}$ & ICV (Poland) \\
\hline
\end{tabular}

\section{Заключение}

На примере гиперкубических вычислительных систем в работе исследованы топологические аспекты масштабируемости параллельных ВС и решаемых на них задач. Предложены и формализованы функции предельного параллелизма и топологической масштабируемости гиперкубических параллельных систем на задачах с полносвязной, кольцевой и звездной топологиями. Приведены графики соответствующих функций, и дан их анализ.

Результаты работы могут быть полезными в сопоставлении топологий параллельных систем с целью оптимизированного под ориентацию системы или под набор решаемых на ней задач их выбора.

\section{References:}

1. Amdahl GM (1967) Validity of the singleprocessor approach to achieving large scale computing capabilities // Proc. AFIPS Conference, Atlantic City, N.J., Apr. 18-20. AFIPS Press, Reston, Va. - 1967, Vol. 30, pp. 483-485.

2. Makagon D, Syromyatnikov E (2011) Seti dlya superkompyuterov // Otkrytye sistemy, 2011, No. 7, pp. 1-8.

3. Kate Matsudaira (2015) Scalable Web Architecture and Distributed Systems // The Architecture of Open Source Applications, El. Resourse, Available: http://www.aosabook.org/en/distsys.html (Accessed: 10.11.2015).

4. Melentiev VA (2014) Embedding of subsystems limiting length and number of paths between vertexes of computing system graph // Upravlenie bol'simi sistemami, 2014, Issue 47, pp. 212-245.
5. Melentiev V (2015) About topological scalability of computing systems // Upravlenie bol'simi sistemami, 2015, Issue 58 (in the press).

6. Melentiev VA (2015) Limit configuring of subsystems in hypercubic computing systems // Informacionnye tekhnologii i vychislitelnye sistemy, 2015, No. 2, pp. 20-30.

7. Voevodin VV, Voevodin VV Parallelnye vychisleniya // Sankt-Peterburg, 2002, pp. 599.

8. Zykov AA (1987) Osnovy teorii grafov // M. Nauka, 1987. pp. 384.

9. Gergel VP (2010) Vysokoproizvoditelnye vychisleniya dlya mnogoyadernyh mnogoprotsessornyh system. Uchebnoe posobie // Nizhnij Novgorod, NNGU, 2010, pp. 421.

10. Konstantinova EV (2014) Kombinatornye zadachi na grafah Keli: uchebnoe posobie // Novosibirsk, Novosibirskij gos. un-t, 2014, pp. 163. 